\title{
Coativação, espasticidade, desempenho motor e funcional na paralisia cerebral
}

\author{
Maíra Seabra de Assumpção ${ }^{1}$ \\ Elaine Carmelita Piucco ${ }^{2}$ \\ Eliane Castilhos Rodrigues Corrêa ${ }^{3}$ \\ Lílian Gerdi Kittel Ries ${ }^{1}$ \\ ${ }^{1}$ Universidade do Estado de Santa Catarina, Florianópolis, SC, Brasil \\ ${ }^{2}$ Fundação Catarinense de Educação Especial, Florianópolis, SC, Brasil \\ ${ }^{3}$ Universidade Federal de Santa Maria, Santa Maria, RS, Brasil
}

\begin{abstract}
Resumo: O objetivo deste estudo foi verificar a associação entre a coativação muscular durante a marcha, a espasticidade, as habilidades funcionais e a função motora ampla em crianças com paralisia cerebral (PC) e comparar estes parâmetros com os de crianças com desenvolvimento típico (DT). Participaram do estudo 16 crianças com DT e 23 com PC. Os instrumentos clínicos utilizados foram: a Escala Modificada de Ashworth para espasticidade, o Pediatric Evaluation of Disability Inventory para habilidades funcionais, 0 Gross Motor Function Measure para função motora ampla. A ativação dos músculos reto femoral e semitendíneo foi analisada durante o ciclo de marcha por meio do cálculo do Índice de Coativação (IC). As medidas de habilidades funcionais e motricidade ampla apresentaram associação entre si, sendo bons indicadores de alterações motoras. O IC não parece ser um bom parâmetro para indicar alterações na função motora em crianças com PC de severidade mínima.
\end{abstract}

Palavras-chaves: Paralisia Cerebral. Espasticidade Muscular. Marcha.

\section{Coactivation, spasticity, motor and functional performance in cerebral palsy}

\begin{abstract}
The purpose of this study was to identify whether or not there is an association between muscle coactivation during gait, spasticity, functional abilities and gross motor behavior in children with cerebral palsy (CP) and to compare these parameters to those of children with typical development (TD). Sixteen children with TD and 23 children with CP participated of this study. The clinical instruments included: the Modified Ashworth Scale for spasticity, the Pediatric Evaluation of Disability Inventory for functional abilities and the Gross Motor Function Measure for gross motor behaviors. Using coactivation index $(\mathrm{Cl})$, activation of the rectus femoris and semitendinosus muscles was analyzed during the participants'gait cycles. Measures of functional abilities were related to parameters of gross motor behaviors and, therefore, providing appropriate indicators of motor changes. Contrary, the $\mathrm{Cl}$ does not seem to be an appropriate parameter for identifying changes in gross motor behavior in children with CP of minimal severity.
\end{abstract}

Keywords: Cerebral Palsy. Muscle Spasticity. Gait.

\section{Introdução}

Os prejuízos decorrentes da paralisia cerebral (PC) resultam em incapacidades e dificuldades no funcionamento típico (STRAUB; OBRZUT, 2009) que incluem anormalidades no tônus muscular, na postura (DONKER; LEDEBT; ROERDINK; SAYELSBERG; BEEK, 2008), no equilíbrio, na coordenação, diminuição da força e perda do controle motor seletivo (PAPAVASILIOU, 2008). A anormalidade mais freqüente no tônus muscular é a espasticidade (MISCIO; DEL CONTE; PIANCA; COLOMBO; PANIZZA; SCHIEPPATI; PIZANO, 2004). Essa desordem provoca um padrão anormal na reciprocidade da inibição da musculatura antagonista, que gera uma coativação muscular excessiva e reforça o comprometimento funcional (PRIORI;
COGIAMANIAN; SPOSTA-MRAKIC, 2006). A coativação muscular funciona como um dos principais meios pelo qual o sistema nervoso estabiliza a posição de um membro ( $\underline{\text { SUZUKI; }}$ SHILLER; GRIBBLE; OSTRY, 2001). Contudo, uma ativação inadequada prejudica o controle motor, que depende da relação entre a musculatura agonista e antagonista (LAMONTAGNE; RICHARDS; MALOUIN, 2000).

Além do déficit significativo observado na habilidade motora e na capacidade de aprendizagem (VAN ZELST; MILLER; RUSSO; MERCHLAND; ROTTY, 2006), o pobre controle motor seletivo determina evolução desfavorável 
da função motora ampla ${ }^{1}$ (VOORMAN; DALLMEIJER; KNOL; LANKHORST; BECHER, 2007) e interfere na aquisição e no desempenho de habilidades funcionais do dia a dia (MANCINI; FIÚZA; REBELO; MAGALHÃES; COELHO; PAIXÃO; GONTIJO; FONSECA, 2002). O aumento da coativação muscular, a espasticidade, o déficit na função motora ampla e nas habilidades funcionais são sintomas comuns na PC e parecem estar relacionados.

Devido a importância da detecção de transtornos na atividade motora, muitos pesquisadores têm direcionado sua atenção no estudo destes parâmetros e nem sempre esta relação é observada. Estudos mostraram que crianças com PC apresentam maior coativação muscular do que crianças com desenvolvimento típico (DT) (DAMIANO; MARTELLOTA; SULLIVAN; GRANATA; ABEL, 2000, VAN DE WALLE; HALLEMANS; HENDRICKX; WAMBACQ; AERTBELIËN; SEVERINJS; DESLOOVERE, 2009, POON; HUI-CHAN, 2009), mas esta pode não estar correlacionada com a Medida da Função Motora Ampla (GMFM) (DAMIANO et al., 2000). A coativação também foi associada com o reflexo de estiramento, mas este não foi associado aos sinais clínicos da espasticidade (POON; HUI-CHAN, 2009). Com relação à coativação e à espasticidade, alguns pesquisadores relatam baixa correlação entre esses parâmetros (VAN DE WALLE; HALLEMANS; HENDRICKX; WAMBACQ; AERTBELIËN; SEVERINJS; DESLOOVERE, 2009). Há estudos que relataram pouca relação entre os sinais clínicos da espasticidade com as desordens de movimento funcionais (DIETZ; SINKJAER, 2007). Contudo, Tuzson et al. encontraram significativa correlação entre a espasticidade e o GMFM (TUZSON; GRANATA; ABEL, 2003) contradizendo os estudos de Abel, Damiano, Blanco, Conaway, Miller, Dabney, Sutherland, Chambers, Dias, Sarwak, Killian, Doyle, Root, Laplaza, Widmann, e Snyder (2003).

A relação entre a coativação e a espasticidade não é clara, portanto examinar tal relação tornase fundamental para a compreensão da função motora de criança com desenvolvimento típico e atípico. A compreensão do desenvolvimento da ação muscular pode colaborar para 0

\footnotetext{
${ }^{1}$ Atividades motoras que envolvem a ação de grandes grupos
musculares, tais como, rolar, engatinhar, ficar em pé, andar e

${ }^{1}$ Atividades motoras que envolvem a ação de grandes grupos
musculares, tais como, rolar, engatinhar, ficar em pé, andar e correr.
} Motriz, Rio Claro, v.17, n.4, p.650-659, out./dez. 2011 planejamento de estratégias de avaliação e de intervenção mais efetivas. Assim, este estudo tem como objetivo verificar a associação entre a coativação muscular durante a marcha, a espasticidade, as habilidades funcionais $e$ a função motora ampla em crianças com PC, bem como, comparar estes parâmetros com os de crianças com DT.

\section{Métodos}

\section{Participantes}

Participaram do estudo 16 crianças com DT e 23 crianças com diagnóstico de PC espástica, com idade entre sete (7) e quatorze (14) anos, capazes de compreender instrução verbal simples e com marcha independente e com marcha independente nível I ou II de acordo com o Sistema de Classificação da Função Motora Grossa (GMFCS) (ROSEMBAUM; WALTER; HANNA; PALISANO; RUSSEL; RAINA; WOOD; BARTLETT; GALUPPI, 2002). O nível I do GMFCS indica que as crianças foram capazes de andar nos espaços internos e externos e subir escadas sem limitações, realizar habilidades motoras grossas, correr e pular, porém com velocidade, equilíbrio e coordenação reduzidos. $O$ nível II indica que as crianças foram capazes de andar nos espaços internos e externos e subir escadas segurando no corrimão, mas com limitações no andar em superfícies irregulares, inclinadas, em espaços lotados ou restritos, bem como com pouca capacidade nas habilidades grossas, como correr e pular.

Das crianças com DT, oito eram do sexo masculino e oito do sexo feminino e das crianças com PC, treze eram do sexo masculino e dez do sexo feminino, sendo que destas, dezesseis apresentavam hemiparesia e sete diparesia. As características antropométricas das crianças são apresentadas na Tabela 1.

Tabela 1. Média e desvio-padrão dos parâmetros idade, peso e altura das crianças com PC $(n=23)$ e com DT $(n=16)$.

\begin{tabular}{cccc}
\hline & Crianças com PC & Crianças com DT & $\mathbf{P}$ \\
\hline & Média \pm DP & Média \pm DP & \\
$\begin{array}{c}\text { Idade } \\
\text { (anos) }\end{array}$ & $10,63 \pm 2,29$ & $9,99 \pm 2,03$ &, 37 \\
$\begin{array}{c}\text { Peso } \\
\text { (kg) }\end{array}$ & $35,93 \pm 12,49$ & $37,61 \pm 9,49$ &, 65 \\
$\begin{array}{c}\text { Altura } \\
\text { (m) }\end{array}$ & $1,40 \pm 0,16$ & $1,40 \pm 0,13$ &, 89 \\
\hline
\end{tabular}

Teste T para dados independentes

Foram excluídas da pesquisa as crianças com doenças sistêmicas (artrite, artrose e diabetes), 
que faziam uso de medicamentos (analgésicos e antiinflamatórios), uso de toxina botulínica e que realizaram cirurgia ortopédica nos últimos seis meses antes do estudo. Os responsáveis de cada criança foram informados sobre as atividades desenvolvidas, assim como seus objetivos. Para a participação das crianças, um Termo de Consentimento Livre e Esclarecido (TCLE) foi assinado pelos pais ou responsáveis. $O$ estudo foi aprovado pelo Comitê de Ética em Pesquisa (protocolo $n^{\circ}$ 108/2008) da Universidade do Estado de Santa Catarina - UDESC.

\section{Instrumentos do Estudo}

Para a avaliação do grau de espasticidade foi utilizada a Escala Modificada de Ashworth (EMA) (BOHANNON; SMITH, 1987) verificando O momento da amplitude articular da resistência ao movimento através da movimentação passiva da extremidade Para a mensuração da função motora ampla foram utilizadas as dimensões $D$ (em pé) e E (andar, correr e pular) da escala Gross Motor Function Measure (GMFM-88) (RUSSEL; AVERU; ROSENBAUN; RAINA; WALTER; PALISANO, 2000). O instrumento usado para medir a capacidade e o desempenho nas habilidades funcionais foi a parte I da versão brasileira (MANCINI; FIÚZA; REBELO; MAGALHÃES; COELHO; PAIXÃO; GONTIJO; FONSECA, 2002) do Pediatric Evaluation of Disability Inventory - PEDI (HALEY; RACZEK; COSTER; DUMAS; FRAGALA-PINKHAM, 2005), que engloba autocuidado, mobilidade e função social.

A coativação muscular foi avaliada por meio do Eletromiógrafo Miotool USB da empresa Miotec com placa conversora analógico/digital de 14 bits de resolução para uma taxa de aquisição de 2000 $\mathrm{Hz}$ e mínima relação de rejeição de modo comum de $110 \mathrm{~dB}$. Para monitorar os músculos avaliados foram utilizados eletrodos de superfície descartáveis passivos com amplificador da marca Medi-trace Kendall-LTP, modelo Chicopee MA 01022. A coativação muscular foi avaliada durante o ciclo de marcha (período entre o primeiro contato do pé com o solo e o próximo contato do mesmo pé com o solo). O ciclo da marcha foi selecionado a partir de imagens no plano sagital pelo sistema Peak Motus (Peak Performance Technologies Inc., Englewood, EUA), que integra uma câmera de vídeo com taxas de aquisição de $60 \mathrm{~Hz}$ fixada a um tripé e um vídeo cassete SVHS-AG da marca Panasonic. A sincronização foi realizada entre o eletromiógrafo e a câmera de vídeo por meio de um LED (Light Emitting Diode), interligado ao eletromiógrafo e fixo na pista, em local de possível captação pela câmera.

\section{Procedimentos}

Após a apresentação e familiarização ao local do estudo, as crianças foram encaminhadas ao vestiário do laboratório para a colocação de uma vestimenta adequada (short e top), pés descalços e cabelos presos. Um avaliador devidamente treinado realizou as mensurações antropométricas na criança (peso e altura) e a aplicação das dimensões D (em pé) e E (andar, correr e pular) do teste da função motora ampla (GMFM-88). Um outro avaliador, por meio de entrevistas com os pais e responsáveis, aplicou a parte I do PEDI para verificar as habilidades funcionais e preencheu a anamnese. $\mathrm{Na}$ sequência foi realizada a avaliação clínica da espasticidade dos membros inferiores (EMA). A mensuração do grau de espasticidade foi realizada com a criança posicionada em decúbito ventral sobre uma maca, na qual foi realizada a movimentação passiva da articulação do joelho em flexão e extensão.

Para o registro do sinal eletromiográfico (EMG) do membro mais acometido (definido pelo maior grau de espasticidade), primeiramente foi feito o procedimento da localização dos ventres musculares para colocação dos eletrodos, sendo os músculos avaliados, reto femoral e semitendíneo, por meio de uma prova de função muscular. A localização e a colocação dos eletrodos foi determinada respeitando as recomendações SENIAM (Surface Electromyography for the Non-Invasive Assessment of Muscles) (HERMENS; FRERIKS; DISSELHORST-KLUG; RAU, 2000). A impedância elétrica da pele foi diminuída limpando-se o local com álcool (70\%). Os eletrodos foram posicionados no reto femoral a $50 \%$ da linha da espinha ilíaca ântero-superior até parte superior da patela e, no semitendíneo a $50 \%$ da linha entre a tuberosidade do ísquio e o epicôndilo medial da tíbia, ambos mantendo uma distância intereletrodos de $2 \mathrm{~cm}$. O eletrodo de referência foi colocado sobre 0 processo espinhoso de C7. A seguir, a criança foi solicitada a caminhar em uma pista em velocidade habitual, com braços soltos ao longo do corpo por pelo menos 10 segundos. Durante a aquisição eletromiográfica foram obtidas imagens 
bidimensionais da marcha para frente do lado mais acometido, considerando cinco tentativas válidas.

\section{Processamento e Análise dos dados}

Por meio do software Ariel Performance Analysis System (APAS), um ciclo da marcha em cada tentativa foi selecionado e a partir da sincronização entre 0 eletromiógrafo e a câmera da vídeo foi calculado o período da atividade muscular correspondente. A análise do ciclo da marcha foi realizada por meio do cálculo do índice de coativação (IC) utilizando o software MATLAB (Versão 5.3 The MathWorks Inc.). O sinal EMG bruto do ciclo da marcha foi filtrado com filtro passa-alta de $10 \mathrm{~Hz}$ e um filtro passabaixa de $500 \mathrm{~Hz}$, a retificação e filtragem dos sinais foram feitas com uma freqüência de corte de $6 \mathrm{~Hz}$ para obter o envoltório linear reduzido a 100 pontos (RMS). Para cada músculo, a normalização dos valores RMS foi calculada da porcentagem da média e do máximo valor RMS obtido em um ciclo de marcha. Optou-se utilizar a normalização pela média do valor RMS do ciclo de marcha, pois obteve o menor coeficiente de variação.

A área comum entre as curvas normalizadas representa a intensidade de ativação muscular simultânea. As duas áreas EMG foram sobrepostas e o IC foi obtido pela razão entre o mínimo valor EMG pelo máximo valor EMG em cada ponto no tempo (o valor mínimo e máximo se alteravam durante o ciclo) e então, fazendo a razão média do período (DAMIANO; MARTELLOTA; SULLIVAN; GRANATA; ABEL, 2000). Se a contração dos dois músculos é simultânea o IC é 100\%. A análise do IC foi realizada sobre a média de cinco ciclos de marcha em cinco tentativas realizada por cada criança.

Os escores totais brutos das habilidades funcionais nas áreas de autocuidado, mobilidade e função social foram resultados da soma de todos os itens pontuados separadamente em cada área de desempenho da parte I do teste. Os dados da medida da função motora ampla foram obtidos a partir da soma dos itens de cada dimensão. Depois dentro de cada uma das dimensões um escore percentual foi calculado (escore da criança/escore máximo da dimensão) $x$ 100. O escore total foi obtido por meio da média aritmética das porcentagens resultantes das dimensões D e E. A pontuação da EMA foi realizada a partir da soma dos resultados obtidos de flexão e extensão de joelho do membro mais acometido.

\section{Análise Estatística}

Os dados descritivos estão relatados como média e desvio-padrão. As variáveis analisadas foram IC, grau de espasticidade, habilidades funcionais nas áreas (autocuidado, mobilidade e função social) e função motora ampla (média das dimensões $\mathrm{D}, \mathrm{E}$ ).

A normalidade dos dados foi verificada por meio do teste de Shapiro-Wilk. Todas as variáveis foram analisadas por meio do Teste $t$ para dados independentes e do Teste de Mann-Whitney para verificar possíveis diferenças entre as crianças com PC e com DT. No grupo com PC, o coeficiente de correlação de Spearmann foi usado para examinar a associação entre as variáveis analisadas. O programa estatístico utilizado foi o Statistical Package for the Social Sciences (SPSS) versão 17.0 para Windows e, para todos os procedimentos, foi adotado o nível de significância de $5 \%(p<0.05)$.

\section{Resultados}

Nas vinte e três crianças PC do estudo, o nível de GMFCS predominante foi o nível I. Verificou-se que dentro desse nível quinze crianças apresentaram hemiparesia e uma diparesia. Já no nível II do GMFCS, foram classificadas sete crianças. Destas, duas crianças apresentaram hemiparesia e cinco diparesia. Em relação ao grau de espasticidade (EMA), em uma criança foi verificado tônus normal (0), dezessete crianças com hipertonia leve (1 a 1,5), quatro com hipertonia moderada (2) e uma criança com hipertonia intensa (3).

A Tabela 2 apresenta os valores médios e desvio padrão das variáveis IC, espasticidade, habilidades funcionais e função motora ampla das crianças com PC e das crianças com DT. O teste $t$ de Student sugere que não há diferença significante no IC entre os grupos $(p \geq 0,05)$. Em relação as variáveis clínicas, todas apresentaram diferença significante entre os grupos avaliados $(p \leq 0,05)$. 
Tabela 2. Média e Desvio Padrão do Índice de Coativação (IC\%) e das variáveis clínicas grau de espasticidade (EMA), habilidades funcionais (PEDI) e a função motora ampla (GMFM) das crianças com PC $(n=23)$ e das crianças com DT $(n=16)$.

\begin{tabular}{lccc}
\hline & Crianças com PC & Crianças com DT & P \\
\hline IC \% $^{\text {a }}$ & $48,02 \pm 7,71$ & $52,11 \pm 7,00$ & 0,10 \\
EMA $^{\text {b }}$ & $1,15 \pm 0,80$ & - & 0,00 \\
PEDI & & $72,69 \pm 0,70$ & 0,00 \\
AutoCuidado $^{\text {b }}$ & $52,48 \pm 7,13$ & $59,00 \pm 0,00$ & 0,00 \\
Mobilidade $^{\mathrm{b}}$ & $58,26 \pm 6,51$ & $64,25 \pm 2,24$ & 0,00 \\
FunçãoSocial $^{\mathrm{b}}$ & $86,91 \pm 9,69$ & $99,47 \pm 0,31$ & 0,00 \\
GMFM total $^{\mathrm{b}}$ & &
\end{tabular}

${ }^{\mathrm{a}}$ Teste T para dados independentes; ${ }^{\mathrm{b}}$ Teste de Mann-Whitney

Entre as crianças com PC, a espasticidade (EMA) e o IC mostraram associação positiva moderada entre si por meio do coeficiente de correlação de Spearman, porém, o mesmo não ocorreu entre ambas as variáveis com as habilidades funcionais e a função motora ampla. Houve associação positiva moderada entre 0
GMFM e as áreas de autocuidado e mobilidade do PEDI. Como era esperado houve associação positiva entre as áreas do PEDI; a mobilidade foi associada moderadamente com autocuidado e função social. Houve associação alta entre auto cuidado e função social (Tabela 3).

Tabela 3. Correlação entre o índice de coativação (IC) e as variáveis clínicas grau de espasticidade (EMA), habilidades funcionais (PEDI) e a função motora ampla (GMFM) das crianças com PC ( $n=23)$.

\begin{tabular}{lccccc} 
& \multirow{2}{*}{ EMA } & \multicolumn{3}{c}{ PEDI } & \multirow{2}{*}{ GMFM } \\
\cline { 3 - 5 } & & Auto Cuidado & Mobilidade & Função Social & \\
\hline IC & $0,62^{* *}$ & 0,14 & 0,17 & 0,16 & 0,05 \\
EMA & & $-0,08$ & $-0,09$ & $-0,06$ & $-0,29$ \\
PEDI & & & $0,68^{* *}$ & $0,74^{* *}$ & $0,45^{* *}$ \\
Auto Cuidado & & & $0,59^{* *}$ & $0,55^{* *}$ \\
Mobilidade & & & & 0,36 \\
Função Social & & & & \\
\hline
\end{tabular}

Correlação de Spearman; * $p<0,05 ;{ }^{* *} p<0,01$.

\section{Discussão}

A PC é uma desordem caracterizada pela presença de espasticidade e menor desempenho nas habilidades funcionais e na função motora ampla. No presente estudo, conforme o esperado observou-se significante diferença entre crianças com PC e com DT em todas as avaliações clínicas, o que demonstra que esses parâmetros são confiáveis para identificar e quantificar possíveis diferenças entre os grupos. A EMA, o GMFM e o PEDI se mostraram sensíveis à detecção de alterações clinicamente importantes. Notou-se diferenças entre os grupos no que se referiu as pontuações alcançadas nas habilidades funcionais e na motricidade ampla, o que não se refletiu no IC.

A coativação da musculatura antagonista é observada no início do aprendizado motor e está intimamente ligada às exigências específicas da tarefa, como direção e força, o que constitui uma parte central dos meios pelos quais o sistema nervoso regula o movimento (DARAINY; OSTRY,
2008). Há estudos que observaram que as crianças com PC utilizam mais freqüentemente a coativação durante a marcha (DAMIANO; MARTELLOTA; SULLIVAN; GRANATA; ABEL, 2000; VAN DE WALLE; HALLEMANS; HENDRICKX; WAMBACQ; AERTBELIËN; SEVERINJS; DESLOOVERE, 2009) e durante o esforço isométrico máximo (DAMIANO; MARTELLOTA; SULLIVAN; GRANATA; ABEL, 2000; POON; HUI-CHAN, 2009). Contudo, os resultados encontrados em nosso estudo mostram que crianças com DT apresentaram um índice maior, porém sem significância estatística. Este resultado pode ser justificado pelo fato das crianças com PC avaliadas, quando comparadas ao grupo DT, não apresentarem um grande déficit na sua locomoção. A maioria das crianças com PC estava classificada no nível I do GMFCS, apresentavam marcha de forma independente e sem a necessidade de aparelhos auxiliares. Nos estudos citados (DAMIANO; MARTELLOTA; SULLIVAN; GRANATA; ABEL, 2000; POON; HUICHAN, 2009), as crianças com PC eram mais 
comprometidas que 0 grupo PC de nossa pesquisa, pois a maioria necessitava de assistência para deambular ou utilizavam aparelhos auxiliares de locomoção.

A pesquisa de Poon e Hui-Chan (2009), com crianças com PC sugere que a transformação de fibras do tipo II para o tipo I pode ser acompanhada por um aumento na sensibilidade ao reflexo de estiramento nos músculos espásticos (POON; HUI-CHAN, 2009). Crianças com PC apresentam mudanças na distribuição das fibras musculares com aumento no diâmetro e na quantidade de fibras do tipo I e deficiência de fibras do tipo II, contudo esta variação está relacionada a severidade e duração da espasticidade (ITO; ARAKI; TANAKA; TASAKI; CHO; YAMAZAKI, 1996). As crianças avaliadas no presente estudo eram independentes para a marcha, níveis I ou II do GMFCS e a maioria apresentava um quadro de hipertonia leve, ou seja, a espasticidade não era severa. Portanto, estas crianças apresentavam uma paralisia cerebral de severidade mínima sugerindo que não houveram mudanças na distribuição das fibras musculares. Acredita-se que crianças PC com maior comprometimento motor e funcional apresentem maior coativação durante a marcha. Os possíveis efeitos negativos de uma excessiva ativação muscular incluem grande ativação muscular total durante a produção da força e alterações na qualidade e quantidade de movimento devido ao acréscimo na rigidez articular (DAMIANO; MARTELLOTA; SULLIVAN; GRANATA; ABEL, 2000).

Foi sugerido que a maior coativação durante a marcha pode ser causada por uma estratégia compensatória para aumentar a estabilidade articular e limitar os graus de liberdade (DAMIANO; MARTELLOTA; SULLIVAN; GRANATA; ABEL, 2000) ou pelo déficit de equilíbrio da crianças com PC (VAN DE WALLE; HALLEMANS; HENDRICKX; WAMBACQ; AERTBELIËN; SEVERINJS; DESLOOVERE, 2009).

A fraqueza muscular também está associada ao aumento da coativação e contribui para o déficit motor (POON; HUI-CHAN, 2009). É importante notar que nem toda coativação dos músculos antagonistas durante um movimento é desnecessária. A coativação é considerada um indicador da capacidade de realização de uma habilidade motora e nas situações em que o músculo agonista está ativado além do nível necessário para estabilidade, o movimento pode ser produzido com sucesso, contudo o custo metabólico do movimento será elevado (FROST; DOWLING; DYSON; BAR-OR, 1997). A coativação pode fazer parte do processo subjacente ao controle motor usado pelo sistema nervoso central para controlar o movimento.

Entre a espasticidade e o IC muscular de PCs, verificou-se uma correlação positiva moderada. Quanto maior espasticidade, maior é a coativação muscular, resultado semelhante a outro estudo que observou correlação baixa mas significativa (VAN DE WALLE; HALLEMANS; HENDRICKX; WAMBACQ; AERTBELIËN; SEVERINJS; DESLOOVERE, 2009). A avaliação da espasticidade, clinicamente ou por meio da resposta do reflexo de estiramento (resposta $M$ ), e das mudanças das propriedades biomecânicas dos músculos podem contribuir para 0 entendimento da hipertonia da criança com PC (POON; HUI-CHAN, 2009). Crianças com espasticidade apresentam uma mudança na distribuição dos tipos de fibras relacionada à severidade do tônus (ITO; ARAKI; TANAKA; TASAKI; CHO; YAMAZAKI, 1996).

Contudo, apesar do IC mostrar associação com a espasticidade em crianças com PC, O IC não diferenciou crianças com $\mathrm{PC}$ de crianças com DT, sem alterações no tônus muscular. Estes resultados podem indicar que $\mathrm{O} I \mathrm{IC}$ em crianças menos comprometidas (a maioria das crianças avaliadas estava no nível I do GMFCS), não é um bom parâmetro para detectar alterações na atividade muscular. Como o IC aumentou com o aumento da espasticidade supõe-se então que este parâmetro seja sensível para detectar diferenças entre crianças DT e crianças PC mais comprometidas.

Em relação às variáveis clínicas, os escores do GMFM não foram correlacionados com os do IC muscular corroborando os resultados de Damiano, Martellota, Sullivan, Granata e Abel (2000) que avaliaram a atividade eletromiográfica dos músculos reto femoral e bíceps femoral de crianças com PC. Também não foi encontrada associação entre o IC e os escores do PEDI, assim como entre os escores da EMA e o PEDI, nem com o GMFM. Estes resultados corroboraram os de Abel, Damiano, Blanco, Conaway, Miller, Dabney, Sutherland, Chambers, Dias, Sarwak, Killian, Doyle, Root, Laplaza, 
Widmann e Snyder (2003), que não encontraram associação entre a EMA e o GMFM. A avaliação clínica da espasticidade por meio da EMA não mostrou ser um bom indicador do comprometimento da criança.

Contudo, Ostensjo, Carlberg e Vollestad (2004) encontraram uma associação positiva moderada entre os escores da EMA e GMFM e com as áreas do PEDI em diferentes grupos de $\mathrm{PC}$, dos quais grande parte apresentava diplegia e a maioria não se enquadrava nos níveis I e II do GMFCS. Essa associação positiva pode ser questionada, já que significaria dizer que crianças com maior espasticidade apresentariam melhores escores na função motora ampla e nas habilidades funcionais. Acredita-se que a direção negativa dessa associação foi omitida já que 0 estudo demonstrou que as crianças mais comprometidas apresentavam maior espasticidade.

As associações verificadas entre o GMFM e as áreas de autocuidado e mobilidade do PEDI mostraram-se positivas e moderadas, assim como entre as áreas do PEDI. A correlação entre - GMFM com as áreas de mobilidade, autocuidado e função social demonstram que a função motora ampla é um ótimo indicador para essas habilidades funcionais (OSTENSJO; CARLBERG; VOLLESTAD, 2004), assim como o GMFCS é para a vida diária (OSTENSJO; CARLBERG; VOLLESTAD, 2003). O déficit em realizar atividades cotidianas, que visam a independência, pode representar uma incapacidade de crianças com PC em atingir seu potencial máximo na função motora ampla (VERSCHUREN; KETELAAR; GORTER; HELDERS; TAKKEN, 2009).

Fatores relacionados com as habilidades funcionais, função motora ampla e coativação muscular são determinantes no planejamento de intervenções e no conhecimento de como a criança interage na sociedade (BECKUNG; HAGBERG, 2002). A diminuição da coativação pode indicar maior habilidade na locomoção humana com maior controle da força e do equilíbrio, indicando neuromaturação (OKAMOTO; OKAMOTO; ANDREW, 2003).

O controle motor é resultado de uma interação contínua entre musculatura, órgãos sensoriais e sistema nervoso central (STIENEN; SCHOUTEN; SCHUURMANS; VAN DER HELM, 2007) e utiliza inicialmente a coativação para aumentar a estabilidade durante um movimento e reduz a coativação quando reconhece uma melhoria dessa (MILNER, 2004). Sugere-se a avaliação da associação desses parâmetros em crianças com maior comprometimento motor e em diferentes fases da marcha.

\section{Conclusão}

Os resultados do presente estudo indicam que crianças com PC, apesar de pouco comprometidas, apresentam maior espasticidade e menor capacidade nas habilidades funcionais e motricidade ampla em relação a crianças com DT, o que mostra que são parâmetros sensíveis para detectar alterações motoras. Já a coativação muscular não parece ser um bom parâmetro para indicar alterações na função motora em crianças com PC de severidade mínima, uma vez que não diferenciou os dois grupos e não foi correlacionada com as habilidades funcionais $e$ com a motricidade ampla.

\section{Referências}

ABEL, M. F.; DAMIANO, D. L.; BLANCO, J.; CONAWAY, M.; MILLER, F.; DABNEY, K.; SUTHERLAND, K.; CHAMBERS, H.; DIAS, L.; SARWAK, J.; KILLIAN, J.; DOYLE, S.; ROOT, L.; LAPLAZA, J.; WIDMANN, R.; SNYDER, B.

Relationship among musculoskeletal impairments and functional health status in ambulatory cerebral palsy. Journal of Pediatric Orthopaedics, New York, v. 23, n. 4, p. 535-541, 2003. Disponível em: $<$ http://dx.doi.org/10.1097/00004694-20030700000022>. Acesso em: 16 out. 2010.

BECKUNG, E.; HAGBERG, G. Neuroimpairments, activity limitations, and participation restrictions in children with cerebral palsy. Developmental Medicine Child Neurology, London, v. 44, n. 5, p. 309-316, 2002. Disponível em: $<$ http://dx.doi.org/10.1111/j.14698749.2002.tb00816.x>. Acesso em: 16 out. 2010.

BOHANNON, R. W.; SMITH, M. B. Inter reliability of a modified Ashworth scale of muscle spasticity. Physical Therapy, New York, v. 67, n. 2 , p. 206207, 1987. Disponível em: $<$ http://physicaltherapyjournal.org/content/67/2/206 full.pdf+html>. Acesso em: 16 out. 2010.

DAMIANO, D. L.; MARTELLOTA, T. L.; SULLIVAN, D. J.; GRANATA, K. P.; ABEL, M. F. Muscle force production and functional performance in spastic cerebral palsy: relationship of cocontraction. Archives Physical Medicine 
Rehabilitation, Chicago, v. 81, n. 7, p. 895-900, 2000. Disponível em:

$<$ http://dx.doi.org/10.1053/apmr.2000.5579>.

Acesso em: 16 out. 2010.

DARAINY, M.; OSTRY, D. Muscle cocontraction following dynamics learning. Experimental Brain Research, Berlin, v. 190, n. 2, p. 153-163, 2008. Disponível em: <http://dx.doi.org/10.1007/s00221008-1457-y>. Acesso em: 16 out. 2010.

DIETZ, V.; SINKJAER, T. Spastic movement disorder: impaired reflex function and altered muscle mechanics. The Lancet Neurology, London, v. 6, p. 725-733, 2007. Disponível em: $<$ http://dx.doi.org/10.1016/S1474-4422(07)70193X>. Acesso em: 16 out. 2010.

DONKER, S. F.; LEDEBT, A.; ROERDINK, M.; SAYELSBERG, G. J. P.; BEEK, P. J. Children with cerebral palsy exhibit greater and more regular postural sway than typically developing children. Experimental Brain Research, Berlin, v. 184, n. 3, p. 363-370, 2008. Disponível em: $<$ http://dx.doi.org/10.1007/s00221-007-1105-y>. Acesso em: 17 out. 2010.

FROST, G.; DOWLING, J.; DYSON, K.; BAR-OR, $O$. Cocontraction in three age groups of children in treadmill locomotion. Journal of

Electromyography and Kinesiology, New York, v. 7, n. 3, p. 179-186, 1997. Disponível em: $<$ http://dx.doi.org/10.1016/S1050-6411(97)846263). Acesso em: 17 out. 2010.

HALEY, S. M.; RACZEK, A. E.; COSTER, W. J.; DUMAS, H. M.; FRAGALA-PINKHAM, M. A. Assessing mobility in children using a computer adaptive testing version of the pediatric evaluation of disability inventory. Archives Physical

Medicine and Rehabilitation, Chicago, v. 86, n. 5, p. 932-939, 2005. Disponível em: $<$ http://dx.doi.org/10.1016/j.apmr.2004.10.032>. Acesso em: 17 out. 2010.

HERMENS, H. J.; FRERIKS, B.; DISSELHORSTKLUG, C.; RAU, G. Development of recommendations for SEMG sensors and sensor placement procedures. Journal of Electromyography and Kinesiology, New York, v. 10, p. 361-374, 2000. Disponível em: $<$ http://dx.doi.org/10.1016/S1050-6411(00)000274>. Acesso em: 17 out. 2010.
ITO, J. I.; ARAKI, A.; TANAKA, H.; TASAKI, T.; $\mathrm{CHO}, \mathrm{K}$.; YAMAZAKI, R. Muscle histopathology in spastic cerebral palsy. Brain \& Development, Tokyo, v. 18, p. 299-303, 1996. Disponível em: $<$ http://dx.doi.org/10.1016/0387-7604(96)00006X >. Acesso em: 17 out. 2010.

LAMONTAGNE, A.; RICHARDS, C. L.; MALOUIN, $F$. Coactivation during gait as an adaptive behavior after stroke. Journal of Electromyography and Kinesiology, New York, v. 10, n. 6, p. 407-415, 2000. Disponível em: $<$ http://dx.doi.org/10.1016/S1050-6411(00)000286). Acesso em: 17 out. 2010.

MANCINI, M. C.; FIÚZA, P. M.; REBELO, J. M.; MAGALHÃES, L. C.; COELHO, Z. A. C.; PAIXÃ̉, M. L.; GONTIJO, A. P. B.; FONSECA, S. T. Comparação do desempenho de atividades funcionais em crianças com desenvolvimento normal e crianças com paralisia cerebral. Arquivos de Neuro-Psiquiatria, São Paulo, v. 60, n. 2B, p. 446-452, 2002. Disponível em: $<$ http://dx.doi.org/10.1590/S0004-

282X2002000300020>. Acesso em: 20 out. 2010.

MILNER, T. E. Accuracy of internal dynamics models in limb movements depends on stability. Experimental Brain Research, Berlin, v. 159, n. 2, p. 172-184, 2004. Disponível em: $<$ http://dx.doi.org/10.1007/s00221-004-1944-8>. Acesso em: 20 out. 2010.

MISCIO, G.; DEL CONTE, C.; PIANCA, D.; COLOMBO, R.; PANIZZA, M.; SCHIEPPATI, M.; PIZANO, F. Botulinum toxin in post-stroke patients: stiffness modifications and clinical implications. Journal of Neurology, Berlin, v. 251, n. 2, p. 189-196, 2004. Disponível em: <http://dx.doi.org/10.1007/s00415-004-0297-3>. Acesso em: 20 out. 2010.

OKAMOTO, T.; OKAMOTO, K.; ANDREW, P. D. Electromyographic developmental changes in one individual from newborn stepping to mature walking. Gait \& Posture, Oxford, v. 14, n. 1, p. 18-27, 2003. Disponível em: $<$ http://dx.doi.org/10.1016/S0966-6362(02)000498>. Acesso em: 20 out. 2010.

OSTENSJO, S.; CARLBERG, E. C.;

VOLLESTAD, N. K. Everyday functioning in young children with cerebral palsy: functional skills, caregiver assistance, and modifications of the environment. Developmental Medicine \& Child Neurology, London, v. 45, n. 9, p. 603-612, 2003. 
Disponível em: <http://dx.doi.org/10.1111/j.14698749.2003.tb00964.x>. Acesso em: 20 out. 2010.

OSTENSJO, S.; CARLBERG, E. B.; VOLLESTAD, N. K. Motor impairments in young children with cerebral palsy: relationship to gross motor function and everyday activities. Developmental Medicine \& Child Neurology, London, v. 46, n. 9, p. 580589, 2004. Disponível em: <http://dx.doi.org/10.1017/S0012162204000994>. Acesso em: 20 out. 2010.

PAPAVASILIOU, A. S. Management of motor problems in cerebral palsy: a critical update for the clinician. European Journal of Paediatric Neurology, London, v. 13, n. 5, p. 387-396, 2008. Disponível em:

$<$ http://dx.doi.org/10.1016/j.ejpn.2008.07.009>. Ac esso em: 20 out. 2010.

POON, D. M. Y.; HUI-CHAN, C. W. Hyperactive stretch reflexes, co-contraction, and muscle weakness in children with cerebral palsy. Developmental Medicine \& Child Neurology, London, v. 51, n. 2, p. 128-135, 2009. Disponível em: <http://dx.doi.org/10.1111/j.14698749.2008.03122.x>. Acesso em: 20 out. 2010.

PRIORI, A.; COGIAMANIAN, F.; SPOSTAMRAKIC, S. Pathophysiology of spasticity. Neurological Science, Amsterdam, v. 27, n. 4, p. 307-309, 2006. Disponível em: <http://dx.doi.org/10.1007/s10072-006-0647-2>. Acesso em: 20 out. 2010.

ROSEMBAUM, P. L.; WALTER, S. D.; HANNA, S. E.; PALISANO, R. J.; RUSSEL, D. J.; RAINA, P.; WOOD, E.; BARTLETT, D. J.; GALUPPI, B. E. Prognosis for gross motor function in cerebral palsy: creation of motor development curves. JAMA, Chicago, v. 288, n. 11, p. 1357-1363, 2002. Disponível em:

<http://dx.doi.org/10.1001/jama.288.11.1357>. Acesso em: 20 out. 2010.

RUSSEL, D. J.; AVERU, L. M.; ROSENBAUN, P. L.; RAINA, P. S.; WALTER, S. D.; PALISANO, R. Improved scaling of the gross motor function measure for children with cerebral palsy: evidence of reliability and validity. Physical Therapy, New York, v. 80, n. 9, p. 873-885, 2000. Disponível em: $<$ http://ptjournal.net/content/80/9/873.full.pdf+html >. Acesso em: 16 out. 2010.

STIENEN, A. H.; SCHOUTEN, A. C.; SCHUURMANS, J.; VAN DER HELM, F. C. T.
Analysis of reflex modulation with a biologically realistic neural network. Journal of

Computational Neuroscience, Boston, v. 23, n. 3, p. 333-338, 2007. Disponível em: <http://dx.doi.org/10.1007/s10827-007-0037-7>. Acesso em: 20 out. 2010.

STRAUB, K.; OBRZUT, J. Effects of cerebral palsy on neuropsychological function. Journal of Developmental and Physical Disabilities, New York, v. 21, n. 9, p. 153-167, 2009. Disponível em: $<$ http://dx.doi.org/10.1007/s10882-009-9130-3>.

Acesso em: 16 out. 2010.

SUZUKI, M.; SHILLER, D. M.; GRIBBLE, P. L.; OSTRY, D. J. Relationship between cocontraction, movement kinematics and phasic muscle activity in single-joint arm movement. Experimental Brain Research, Berlin, v. 140, n. 2, p. 171-181, 2001. Disponível em: <http://dx.doi.org/10.1007/s002210100797>. Acesso em: 16 out. 2010.

TUZSON, A. E.; GRANATA, K. P.; ABEL, M. F. Spastic velocity threshold constrains functional performance in cerebral palsy. Archives of Physical Medicine and Rehabilitation, Chicago, v. 84, p. 1363-1368, 2003. Disponível em: <http://dx.doi.org/10.1016/S0003-9993(03)001990) . Acesso em: 16 out. 2010.

VAN DE WALLE, P.; HALLEMANS, A.; HENDRICKX, C.; WAMBACQ, H.; AERTBELIËN, E.; SEVERINJS, D.; DESLOOVERE, K. Are excessive cocontractions during walking in children with cerebral palsy caused by spasticity? Gait \& Posture, Oxford, v. 30S, p. S16-S17, 2009. Disponível em: <http://dx.doi.org/ 10.1016/j.gaitpost.2009.08.027>. Acesso em: 16 out. 2010.

VAN ZELST, B. R.; MILLER, M. D.; RUSSO, R. N.; MERCHLAND, S.; ROTTY, S. Activities of daily living in children with hemiplegic cerebral palsy: a cross-sectional evaluation using the assessment of motor and process skills. Developmental Medicine \& Child Neurology, London, v. 48, n. 9, p. 723-727, 2006. Disponível em:

<http://dx.doi.org/10.1017/S0012162206001551>. Acesso em: 16 out. 2010.

VERSCHUREN, O.; KETELAAR, M.; GORTER, J, W.; HELDERS, P. J. M.; TAKKEN, T. Relation between physical fitness and gross motor capacity in children and adolescents with cerebral palsy. Developmental Medicine \& Child Neurology, 
London, v. 51, n. 11, p. 866-871, 2009. Disponível em: <http://dx.doi.org/10.1111/j.1469-

8749.2009.03301.x>. Acesso em: 16 out. 2010.

VOORMAN, J. M.; DALLMEIJER, A. J.; KNOL, D. L.; LANKHORST, G. J.; BECHER, J. G.

Prospective longitudinal study of gross motor function in children with cerebral palsy. Archives of Physical Medicine and Rehabilbitation, Chicago, v. 88, n. 7, p. 871-877, 2007. Disponível em:

<http://dx.doi.org/10.1016/j.apmr.2007.04.002>. Acesso em: 16 out. 2010.

Este estudo foi anteriormente apresentado no evento III Congresso Internacional de Especialidades Pediátricas em Curitiba e foi classificado como segundo melhor trabalho na qualidade de tema livre-oral (fisioterapia).

Endereço:

Lílian Gerdi Kittel Ries

Rua Pascoal Simone, 358 Coqueiros

Florianópolis SC Brasil

88080-350

Telefone: (48) 3321.8600

Fax: (48) 3321.8607

e-mail: d2lgkr@udesc.br liliangkr@yahoo.com.br

Recebido em: 17 de outubro de 2010.

Aceito em: 22 de agosto de 2011.

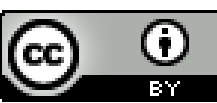

Motriz. Revista de Educação Física. UNESP, Rio Claro, SP, Brasil - elSSN: 1980-6574 - está licenciada sob Creative Commons - Atribuição 3.0 